\title{
Strategies to control pertussis in infants
}

\author{
Gayatri Amirthalingam
}

\section{Correspondence to}

Dr Gayatri Amirthalingam, Immunisation, Hepatitis and Blood Safety department, Public Health England; gayatri.amirthalingam@phe. gov.uk

Received 20 December 2012 Revised 9 April 2013 Accepted 24 April 2013 Published Online First 22 May 2013

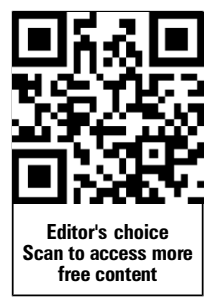

To cite: Amirthalingam $\mathrm{G}$. Arch Dis Child

2013;98:552-555.

\section{ABSTRACT}

The UK is currently in the midst of a large outbreak of pertussis, with the highest morbidity and mortality occurring in young unimmunised infants. This review considers the potential strategies to optimise control of pertussis in infants, including vaccination of (1) adolescents, (2) close household contacts of newborn infants (cocooning), (3) newborn infants and

(4) pregnant women. The paper discusses the evidence base for each of these strategies and considers the rationale for the recent introduction of a temporary vaccination programme for pregnant women in the UK in response to the ongoing outbreak.

\section{BACKGROUND AND RECENT EPIDEMIOLOGY}

High vaccine coverage, the introduction of an accelerated infant schedule, and the inclusion of pertussis in the preschool booster have contributed to a major overall decline in incidence of pertussis between 1998 and 2009. ${ }^{1-3}$ Despite this, pertussis remains the most common cause of hospitalisation and death in infants from a disease that is potentially preventable through the current UK routine vaccination programme, ${ }^{3}$ and continues to display 3-4 yearly cyclical peaks in activity. This suggests that while pertussis vaccines are effective in preventing clinically apparent disease, they are less effective in preventing Bordetella pertussis infection and transmission.

Many countries with longstanding vaccination programmes have experienced recent increases in adolescent and adult disease, attributed to improved case ascertainment and waning immunity following vaccination. ${ }^{4-6}$ Since mid-2011, the UK has been experiencing a national outbreak of pertussis, predominantly affecting adolescents and adults. $^{7}{ }^{8}$ Heightened activity continued in 2012 extending to very young infants, who are at highest risk of serious complications and death. ${ }^{8-10}$ Although the overall incidence remains low compared with the resurgence in the 1970s, the marked rise in incidence among infants less than 3 months of age (258 per 100000 in 2012 compared with 101 per 100000 population in the last peak year, 2008) (figure 1) led to the declaration of a national outbreak by the Health Protection Agency in April 2012. ${ }^{11}$ An urgent review by the Joint Committee on Vaccination and Immunisation (JCVI) culminated in the announcement of a temporary immunisation programme for pregnant women in the UK as an outbreak response. ${ }^{12}$ This paper considers the potential strategies for optimising pertussis control with the main focus on preventing infant deaths.

\section{Adolescent pertussis booster}

Reported increases in pertussis incidence among adolescent and adult populations have led some countries including the USA, Australia and France to introduce a routine adolescent pertussis booster. In 2010, a WHO review of pertussis control strategies concluded that such boosters do prevent pertussis in adolescents and adults, but that there is insufficient evidence of indirect protection for young infants. ${ }^{13}$ Since the US recommended the use of Tdap for adolescents in 2006, coverage among 13-17-year-olds has slowly increased from $10.8 \%$ in 2006 to $78.2 \%$ in $2011 .^{14}$ Over this time, incidence in adolescents aged 11-18 years has declined, but there has been no discernible impact on infant disease. ${ }^{15}$ This may be partly attributable to the suboptimal coverage, but also may reflect contact patterns between adolescent and infant populations. Studies of social mixing suggest that adolescents tend to interact primarily with other adolescents, and have less contact with very young infants. ${ }^{16}{ }^{17}$ Similarly, modelling and cost effectiveness evaluation in the UK in 2001 suggested little impact of an adolescent booster on infant pertussis. ${ }^{2}$ Recent data from Australia suggested some benefit for infants from a one-off school-based catch-up programme (with a broad age group from 12 to 19 years) followed by a routine programme targeting adolescents aged 12 years. ${ }^{18}$

Data on the duration of protection from an adolescent pertussis booster are limited, with predicted estimates of between 6 and 10 years. ${ }^{19}$ Extrapolation from an adolescent trial predicted that the various pertussis antibodies would reach prevaccination rates between 9.5 and 15 years after the dose of dTap. ${ }^{20}$ Waning immunity following an adolescent booster, has the potential to leave adults of child-bearing age susceptible. A resulting shift in disease to population groups who are a more frequent source of infection to infants, ${ }^{21-23}$ has led to calls for decennial boosting with pertussis to supplement current dT schedules in some countries. ${ }^{24}$ In the UK, where adult boosters are not used, modelling the impact of an adolescent booster is required to predict the impact on infant disease in the long term, and this is likely to influence the cost effectiveness of such an approach.

\section{Cocooning strategy}

The rationale for a 'cocooning strategy' (vaccinating close household contacts of young infants, including postpartum women) is based on evidence that infants often acquire pertussis from a household contact. ${ }^{21-23}$ A study conducted in Canada, France, Germany and the USA showed that when pertussis occurred in infants, where a source was identified, household members-primarily parents-were the potential source of $B$. pertussis in $76-83 \%$ of cases. ${ }^{22}$ In a US study of infants with pertussis, in the $43 \%$ cases where the source was identified, over $70 \%$ had been infected by their 


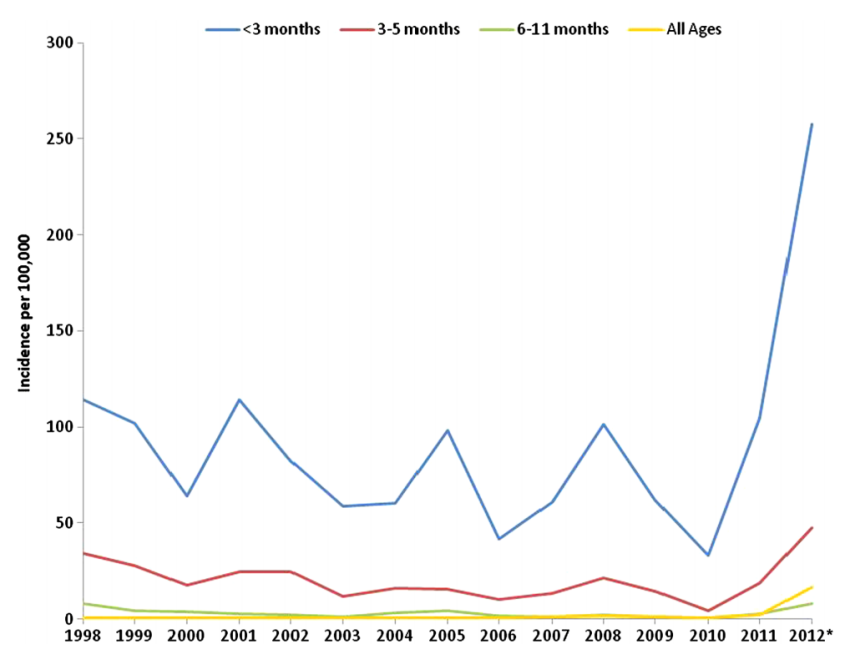

Figure 1 Incidence of laboratory-confirmed pertussis, by total case-patients in infants $<1$ year and all ages in England and Wales, 1998-week 472012.

mother or other family member, the majority of whom were aged 20 years or more. ${ }^{23}$ In a UK study of infants admitted to paediatric intensive care with respiratory complications, $20 \%$ had laboratory evidence of pertussis and half of these were probably infected from an adult family member. ${ }^{25}$

For postpartum vaccination of mothers to be effective, by preventing transmission to the infant, mothers should be protected very rapidly after delivery. ${ }^{26}$ Moreover, where infants have acquired pertussis from their mother, onset of maternal symptoms has been reported to occur before delivery in a fifth of cases. ${ }^{27}$ Studies of antibody responses to Tdap in postpartum women have shown that Immunoglobulin A and Immunoglobulin $G$ responses to pertussis antigens are not detectable until 5-7 days postvaccination, and the maximum response is not achieved until 14 days after vaccination. $^{28}$ This leaves a 2-week delay in 'potential protection' for the infant during a period of high vulnerability. ${ }^{22}$

The cocooning strategy has been recommended in some countries including Australia, US and France. The 2010 SAGE review concluded that while such a strategy may help to protect newborns, data on the implementation and impact at a population level were limited. ${ }^{13}$ The cocooning strategy has been difficult to implement due to the lack of a platform for postpartum administration of vaccines and programmatic issues in administering vaccine to fathers and other adult household contacts. ${ }^{29}$

\section{Neonatal immunisation}

Pertussis immunisation at birth has been proposed following the demonstration of immune responses to pertussis in neonates. ${ }^{30}{ }^{31}$ However, the lack of a serological correlate of protection poses a significant challenge in determining the clinical efficacy of this approach. An early study using a birth dose of DTaP demonstrated lower pertussis antibody levels after completion of the primary schedule compared with the control group. ${ }^{32}$ Despite the absence of a licensed monovalent pertussis vaccine for neonatal use, more recent studies using a birth dose of a monovalent acellular pertussis (aP) vaccine showed high levels of antibodies against pertussis antigens during the first 2 months of life with no decline in pertussis antibody levels following completion of the primary schedule. However, two of these studies did demonstrate interference with the responses to Haemophilus influenzae type $\mathrm{b}$ and hepatitis $\mathrm{B}$ antigens. ${ }^{31} 33$ The exact mechanism for this interference is not known, although bystander T-lymphocyte interference has been proposed as one possibility. ${ }^{34}$ Further investigation of neonatal immunisation is required.

Improving the timeliness of the primary infant schedule/ commencing primary infant course at 6 weeks

Timely primary infant immunisation is important in protecting infants at the earliest opportunity. Vaccine effectiveness of a single dose of pertussis vaccine in infants less than 6 months of age has been estimated at $61.7 \%$ (95\% CI $53.4 \%$ to $68.6 \%$ ) in England and Wales. ${ }^{3}$ Data on the age at which infants born between 1 January 1996 and 1 May 2010 received their first dose of DTaP in England and Wales suggest an improvement in timeliness. Before 2006, almost one-third of infants had not received a single dose of DTaP by 10 weeks age; by 2010 , this had declined to less than one-fifth. While timely administration of the infant schedule is important, data on cases of pertussis confirmed during 2012 show that over 60\% infant cases occurred by 8 weeks of age, prior to eligibility for routine infant vaccination (figure 2 ).

Scheduling of the first dose of DTaP at 6 weeks of age, as is recommended in many countries as part of the Expanded Programme on Immunisation (EPI) schedule, could potentially afford earlier protection to infants. ${ }^{35} 36$ In an Australian study, the potential benefits of acceleration of the first dose was estimated to reduce notifications, hospitalisations and hospital bed days by $8 \%, 9 \%$ and $12 \%$, respectively, with larger reductions in epidemic years. ${ }^{36}$ In a US study, acceleration of the first dose was predicted to result in a $9 \%$ reduction in cases, $9 \%$ reduction in hospitalisations, and $6 \%$ reduction in deaths attributable to pertussis among infants $<3$ months of age. ${ }^{35}$ If accurate, this would translate to 37 fewer cases of the 409 confirmed cases in infants under 3 months of age in the first 47 weeks of 2012 in England and Wales. Adding to the relatively limited impact, this option requires consideration of the impact, in terms of agedependent immunogenicity of other vaccines in the primary infant schedule.

\section{Vaccination of pregnant women}

Vaccinating women in the third trimester of pregnancy offers the opportunity to provide early protection to infants through increased transplacental transfer of maternal antibodies. A number of studies have provided evidence supporting the existence of efficient transplacental transfer of pertussis antibodies. $^{37} 38$ Cord blood from newborn infants whose mothers received Tdap during or before pregnancy had higher

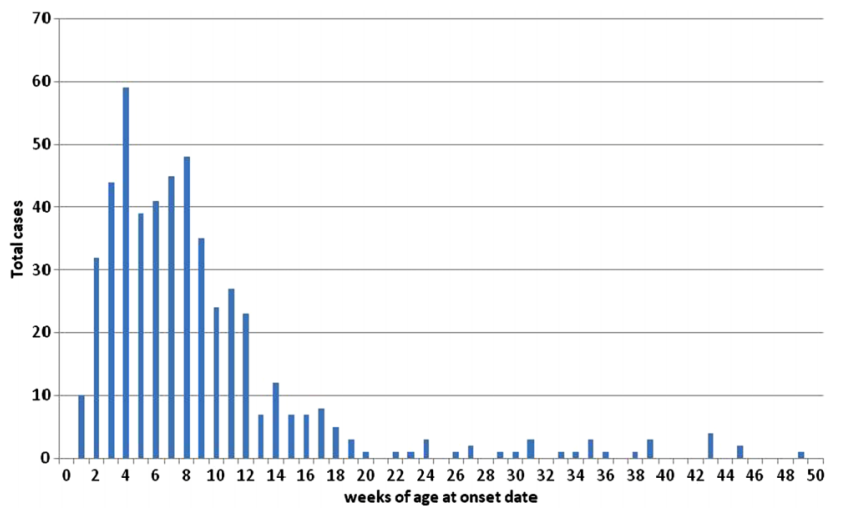

Figure 2 Distribution of confirmed pertussis cases in the first year of life (England and Wales, 2012 up to week 47). 
concentrations of pertussis antibodies when compared with cord blood from newborn infants of unvaccinated mothers. ${ }^{37} 38$ Despite the lack of a serological correlate of protection, this option offers the potential of protecting the infant from birth until active immunity is achieved. ${ }^{39}$ Pertussis-specific secretory IgA has also been demonstrated in breast milk 1-2 weeks after vaccination. ${ }^{40}$ Additionally, vaccination in pregnancy is likely to prevent maternal infection at the time of delivery and exposure of the infant.

Although there is a theoretical risk for severe local reactions, including Arthus reactions for pregnant women receiving multiple doses of tetanus and diphtheria-containing vaccines at closely spaced intervals, recent data has demonstrated the tolerability of repeated doses of tetanus and diphtheria-containing vaccines in adults with intervals as short as 1 month. ${ }^{41}$ Furthermore, large studies on the use of inactivated tetanus-toxoid-containing vaccines in pregnant women have not reported clinically significant severe adverse events, and these vaccines have been extensively used in pregnant women worldwide to prevent neonatal tetanus. ${ }^{42} 43$ Although data on the use of acellular pertussis containing vaccines in pregnant women are limited, a review of reports to the Vaccines Adverse Event Reporting System (VAERS) in the USA did not identify any concerning patterns in maternal, infant or fetal outcomes in women receiving Tdap in pregnancy between 2005 and 2010, ${ }^{44}$ and based on current fertility rates, the majority of women in the UK are likely to require no more than two doses of pertussis vaccine in pregnancy.

In 2011, the USA was the first country to recommend the use of Tdap in pregnant women who previously had not received Tdap in adulthood. ${ }^{45}$ Based on insufficient antibody persistence from preconception or early pregnancy vaccination, this advice has recently been updated to recommend offering women Tdap in every pregnancy in the third trimester. ${ }^{46} 47$

Some studies have suggested that maternal pertussis antibodies can inhibit pertussis-specific antibody responses of infants vaccinated with $\mathrm{DTaP}^{48}{ }^{49}$ The lack of correlates of protection means that the clinical relevance of this 'blunting' effect is uncertain. ${ }^{50}$ Interim trial data from a study comparing immune responses in infants born to mothers vaccinated with either Tdap or $\mathrm{Td}$ in pregnancy, suggested elevated antipertussis antibody levels at birth and before the first dose of infant vaccines, but lower antibody levels after dose 3 in the first group (unpublished ${ }^{51}$ ). The US Advisory Committee on Immunisation Practices (ACIP) concluded that the duration of blunting was short, ${ }^{48}$ and potential risk of shifting the burden to older infants, where the morbidity and mortality was lower, was outweighed by the potential protection afforded by maternal antibodies. ${ }^{50}$

\section{CONCLUSION}

The current outbreak in the UK highlights the overwhelming burden of infant pertussis, in terms of morbidity and mortality that occurs prior to the age of routine vaccination. Maternal immunisation provides the only currently available option for protecting infants right from birth and during the period of highest vulnerability and greatest disease burden. As a consequence, the Department of Health, on the advice of JCVI, has introduced a temporary maternal pertussis immunisation programme to minimise morbidity and prevent further infant deaths while the outbreak continues. Evaluation of this programme, including monitoring vaccine coverage, impact on disease incidence and immune response in infants, vaccine effectiveness and safety, which is currently underway, will be critical in informing the long-term strategy for optimising pertussis control in the UK.
Acknowledgements The author wishes to thank Sonia Ribeiro and Helen Campbell for providing the surveillance data which is included in the paper. The author wishes to thank Dr Mary Ramsay for her input and comments on the manuscript.

Contributors GA was responsible for the concept and design of the manuscript. GA undertook the literature review and drafted the initial submission and revised the manuscript in light of reviewers' comments.

\section{Competing interests None.}

Provenance and peer review Commissioned; externally peer reviewed.

\section{REFERENCES}

1 Joint Committee on Vaccination and Immunisation. JCVI Minutes of the Meeting Held on Monday 9 October 2000. 2000 http://www.dh.gov.uk/prod_consum_dh/ groups/dh_digitalassets/@dh/@ab/documents/digitalasset/dh_117402.pdf

2 Edmunds WJ, Brisson M, Melegaro A, et al. The potential cost-effectiveness of acellular pertussis booster vaccination in England and Wales. Vaccine 2002:20:1316-30.

3 Campbell H, Amirthalingam G, Andrews N, et al. Accelerating the control of pertussis in England and Wales. Emerg Infect Dis 2012;18:38-47.

4 Wendelboe AM, Van Rie A, Salmaso S, et al. Duration of immunity against pertussis after natural infection or vaccination. Pediatr Infect Dis J 2005;24(Suppl 5):S58-61.

5 Cherry JD. The epidemiology of pertussis: a comparison of the epidemiology of the disease pertussis with the epidemiology of Bordetella pertussis infection. Pediatrics 2005:115:1422-7.

6 Klein NP, Bartlett J, Rowhani-Rahbar A, et al. Waning protection after fifth dose of acellular pertussis vaccine in children. N Eng J Med 2012;367:1012-19.

7 Laboratory confirmed cases of pertussis reported to the enhanced pertussis surveillance programme (England and Wales): annual report 2011. Health Protection Report 2012;6 http://www.hpa.org.uk/hpr/archives/2012/hpr0812.pdf

8 Confirmed pertussis in England and Wales: data to end December 2012. Health Protection Report 2013;7 http://www.hpa.org.uk/hpr/archives/2013/news0513. htm\#prtsss

9 Rise in whooping cough cases. Northern Ireland: Public Health Agency. http://www. publichealth.hscni.net/news/rise-cases-whooping-cough

10 Increased levels of pertussis circulating in Scotland, 2012 http://www.hps.scot.nhs. uk/ewr/article.aspx

11 Confirmed cases of pertussis in England continues to increase. Health Protection Report 2012;6 http://www.hpa.org.uk/hpr/archives/2012/news1512.htm\#prtsss

12 "Pregnant women to be offered whooping cough vaccination", 28 September 2012. Department of Health website, http://www.dh.gov.uk/health/2012/09/ whooping-cough/

13 World Health Organization. Pertussis vaccines: WHO position paper. Wkly Epidemiol $\operatorname{Rec}$ 2010;85:385-400.

14 National and state vaccine coverage amongst adolescents aged 13-17 years-United States, 2011. MMWR 2012;61:671-7.

15 Skoff TH, Cohn AC, Clark TA, et al. Early impact of the US Tdap vaccination program on pertussis trends. Arch Pediatri Adolesc Med 2012;166:344-9.

16 Mossong J, Hens N, Jit M, et al. Social contacts and mixing patterns relevant to the spread of infectious diseases. PLoS Med 2008;5:e74

17 Rohani $P$, Zhong $X$, King AA. Contact network structure explains the changing epidemiology of pertussis. Science 2010;330:982-5.

18 Quinn HE, McIntyre PB. The impact of adolescent pertussis immunization, 20042009: lessons from Australia. Bull World Health Organ 2011;89:666-74.

19 Lavine JS, Bjørnstad ON, de Blasio BF, et al. Short-lived immunity against pertussis, age-specific routes of transmission, and the utility of a teenage booster vaccine. Vaccine 2012;30:544-51.

20 Barreto L, Guasparini R, Meekison W, et al. Humoral immunity 5 years after booster immunization with an adolescent and adult formulation combined tetanus, diphtheria and 5-component acellular pertussis vaccine. Vaccine 2007:25:8172-79.

21 Kowalzik F, Barbosa AP, Fernandez VR, et al. Prospective multinational study of pertussis infection in hospitalized infants and their household contacts. Pediatr Infect Dis 2007:26:238-42.

22 Wendelboe AM, Hudgens MG, Poole C, et al. Estimating the role of casual contact from the community in transmission of Bordetella pertussis to young infants. Emerg Themes Epidemiol 2007;4:15

23 Bisgard KM, Pascual FB, Ehresmann KR, et al. Infant pertussis: who was the source? Pediatr Infect Dis J 2004;23:985-9.

24 Zepp F, Heininger U, Mertsola J, et al. Rationale for pertussis booster vaccination throughout life in Europe. Lancet Infect Dis 2011;11:557-70.

25 Crowcroft NS, Booy R, Harrison T, et al. Severe and unrecognised: pertussis in UK infants. Arch Dis Child. 2003:88:802-6.

26 Munoz F, Englund J. Infant pertussis: is cocooning the answer? CID 2011:53:893-6.

27 de Greeff SC, Mooi FR, Westerhof A, et al. Pertussis disease burden in the household: how to protect young infants. Clin Infect Dis 2010;50:1339-45. 
28 Halperin BA, Morris A, MacKinnon-Cameron D, et al. Kineticsa of the antibody response to tetanus-diphtherias-acellular pertussis vaccine (Tdap) in women of child bearing age and post partum women. Clin Infect Dis 2011;53:885-92.

29 Healy CM, Rench MA, Baker CJ. Implementation of cocooning against pertussis in a high-risk population. Clin Infect Dis 2011:52:157-62.

30 Belloni C, De Silvestri A, Tinelli C, et al. Immunogenicity of a three-component acellular pertussis vaccine administered at birth. Pediatrics 2003:111:1042-5.

31 Wood N, Mclntyre P, Marshall $\mathrm{H}$, et al. Acellular pertussis vaccine at birth and one month induces antibody responses by two months of age. Pediatr Infect Dis J 2010;29:209-15.

32 Halasa NB, O'Shea A, Shi JR, et al. Poor immune responses to a birth dose of diphtheria, tetanus, and acellular pertussis vaccine. J Pediatr 2008;153: 327-32

33 Knuf $M$, Schmitt $H J$, Jacquet JM, et al. Booster vaccination after neonatal priming with acellular pertussis vaccine. J Pediatr 2010;156:675-8.

34 Wood N, Siegrist CA. Neonatal immunization: where do we stand? Curr Opin Infect Dis 2011;24:190-5.

35 Shinall MC, Peters TR, Zhu Y, et al. Potential impact of acceleration of the pertussis vaccine primary series for infants. Pediatrics 2008;122:1021-6.

36 Foxwell AR, McIntyre $P$, Quinn $H$, et al. Severe pertussis in infants: estimated impact of first vaccine dose at 6 versus 8 weeks in Australia. Pediatr Infect Dis J 2011;30:161-3.

37 Gall SA, Myers J, Pichichero M. Maternal immunization with tetanus-diphtheria-pertussis vaccine: effect on maternal and neonatal serum antibody levels. Am J Obstet Gynecol 2011;204:334, e1-5.

38 Leuridan E, Hens N, Peeters N, et al. Effect of a prepregnancy pertussis booster dose on maternal antibody titers in young infants. Pediatr Infect Dis $J$ 2011;30:608-10.

39 Van Rie A, Wendelboe AM, Englund JA. Role of maternal pertussis antibodies in infants. Pediatr Infect Dis J 2005;24(5 Suppl):S62-5.

40 Halperin BA, Morris A, Mackinnon-Cameron D, et al. Kinetics of the antibody response to tetanus-diphtheria-acellular pertussis vaccine in women of childbearing age and postpartum women. Clin Infect Dis 2011;53:885-92.
41 Beytout J, Launay 0, Guiso N, et al. Safety of Tdap-IPV given one month after Td-IPV booster in healthy young adults: a placebo-controlled trial. Hum Vaccin 2009;5:315-21.

42 Newell KW, Lehmann D, Leblanc DR, et al. The use of toxoid for the prevention of tetanus neonatorum. Final report of a double-blind controlled field trial. Bull World Health Organ 1966;35:863-71.

43 Schofield FD, Tucker VM, Westbrook GR. Neonatal tetanus in New Guinea. Effect of active immunization in pregnancy. Br Med J 1961;2:785-9.

44 Zheteyeva YA, Moro PL, Tepper NK, et al. Adverse event reports after tetanus toxoid, reduced diphtheria toxoid, and acellular pertussis vaccines in pregnant women. Am J Obstet Gynecol 2012;207:59, e1-7.

45 Updated Recommendations for Use of Tetanus Toxiod, Reduced Diphtheria Toxoid and Acellular Pertussis Vaccine (Tdap) in Pregnant Women and Persons Who Have or Anticipate Having Close Contact with an infant Aged \&lt:12 months-Advisory Committee on Immunization Practices (ACIP), 2011. MMWR 2011;60(41):1424-6.

46 Healy CM, Rench MA, Baker CJ. Importance of timing of maternal Tdap immunization and protection of young infants. Clin Infect Dis 2012. Advanced Access.

47 ACIP provisional updated recommendations on use of tetanus toxoid, reduced diphtheria toxoid and acellular pertussis vaccine (Tdap) for pregnant women, 2012. http://www.cdc.gov/vaccines/recs/provisional/downloads/Tdap-pregnant-Oct-2012.pd

48 Van Savage J, Decker MD, Edwards KM, et al. Natural history of pertussis antibody in the infant and effect on vaccine response. J Infect Dis 1990;161:487-92.

49 Englund JA, Anderson EL, Reed GF, et al. The effect of maternal antibody on the serologic response and the incidence of adverse reactions after primary immunization with acellular and whole-cell pertussis vaccines combined with diphtheria and tetanus toxoids. Pediatrics 1995;96(3 Pt 2):580-4.

50 Centers for Disease Control and Prevention (CDC). Updated recommendations for use of tetanus toxoid, reduced diphtheria toxoid and acellular pertussis vaccine (Tdap) in pregnant women and persons who have or anticipate having close contact with an infant aged $<12$ months -- Advisory Committee on Immunization Practices (ACIP), 2011. MMWR 2011;60:1424-6.

51 Dalhousie University. Pertussis maternal immunization study. Identifier: NCT00553228. http://clinicaltrials.gov/show/nct00553228 (accessed 27 Mar 13). 